\title{
Evolution of Different Music Genres
}

\author{
Selvakumar Kamalnathan, Yash Mishra, Vijay Kumawat, Vaibhav Bangwal
}

\begin{abstract}
This paper aims to study the evolution of five different music genres: Hip-Hop, Rock, Pop, Country and Metal over the last five decades. Each style of music has been classified into three-time slots: Before the 2000s, between 2000 to 2009 and from 2010 to present. Length of lyrics for each genre over the three different time slots has been compared. Similarly, most frequently used words were studied to understand the mood and expression of emotions which each style carries and how they have evolved. Then the lexical diversity and sentimental analysis over time of each music type have been studied to analyze how the songs have evolved to satisfy the ever-changing taste of listeners and the dynamic underlying tone which they carry. We have also studied the various classification models to predict genres with lyrics based on numerous parameters like most frequently words, word- length, lyrics length. At last, we present our opinion on what can we expect from each music-genre in the near future.
\end{abstract}

Keywords-Natural Language Processing, Sentimental Analysis, Music, Lyrics Analysis, Predictive Analysis, Machine Learning, RStudio.

\section{INTRODUCTION}

Lyrics of a song suggest the views and opinions of the artist who writes it, but a genre's words are a reflection of the society and what it wants to hear. Lyrics analysis is a difficult task because of the song's different structure which requires pragmatic and cautious selection of features. Music is omnipresent in our lives and holds power to shape our thoughts and action in a very subtle way. Songs are generally studied based on their music, but the concept of 'Lyrics Analysis' has gained popularity after improvements in various techniques in Natural Language Processing. This work aims to study songs purely based on its lyrics and see how successful is Natural Language Processing in analyzing songs of different genres. The songs are divided into five categories: Pop, Hip-Hop, Metal, Rock and Country and each genre is further divided into three-time slots: Before the 2000s, 2000 - 20009, 2010 - 2019.

Revised Manuscript Received on October 30, 2019.

* Correspondence Author

Dr. Selvakumar Kamalnathan*, Associate Professor, School of Computer Science Engineering, Vellore Institute of Technology (VIT), Vellore (Tamil Nadu), India.

Selvakumar Kamalnathan, Department of Computer Science Engineering, Vellore Institute of Technology (VIT), Vellore (Tamil Nadu), India. E-mail. kselvakumar@vit.ac.in

Yash Mishra, Department of Computer Science Engineering, Vellore Institute of Technology (VIT), Vellore (Tamil Nadu), India. E-mail. yash.mishra2016@vitstudent.ac.in

Vijay Kumawat, Department of Computer Science Engineering, Vellore Institute of Technology (VIT), Vellore (Tamil Nadu), India. Email.vvijaykumawat256@gmail.com.in

Vaibhav Bangwal, Department of Computer Science Engineering, Vellore Institute of Technology (VIT), Vellore (Tamil Nadu), India. Email. vaibhav.bangwal2016@vitstudent.ac.in

(c) The Authors. Published by Blue Eyes Intelligence Engineering and Sciences Publication (BEIESP). This is an open access article under the CC BY-NC-ND license (http://creativecommons.org/licenses/by-nc-nd/4.0/)
Then the length of the lyrics of each genre, frequently used words, lexical diversity and sentimental analysis over time for each style has been performed to understand how each music type has evolved to societies' demands and liking. This study enables us to predict what we can expect from music and how it would influence us in the future. At last, we perform genre classification of songs based on lyrics and present which model, given our parameters, perform best in music genreprediction.

\section{METHOD}

\section{A. Data Selection and Preprocessing}

The aim of this work is to understand how music has evolved over time and how each music genre has changed differently from the rest. This required a dataset with mainly three columns: lyrics, genre and year of release. While there are numerous datasets available which provide the first two columns, we failed to find a dataset which had all three required fields. Therefore, we have used Sam Isenberg's 'Lyrics Classifier dataset' [1] who had provided the Genius [2] song identification number for each song. This enabled us to use 'geniusr library' and run 'get_song_meta' function for

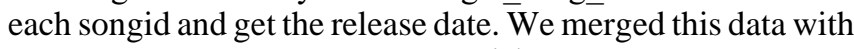
another dataset from Gyanendra Mishra's dataset provided in Kaggle [3] and deleted any duplicate songs. We then manually added songs of each genre so that each time slot had ample number of songs to study from.

Lyrics were cleaned by first converting abbreviations like won't, can't, 'll, 've 'd, 'm etc. to their corresponding formal self. All special character was removed and everything was then converted to lowercase. Redundant words like 'ooh', 'baby', 'yeah' etc. were removed. Now, five new data frames were created for each of the five music genres: Pop, Hip-Hop, Rock, Country, Metal and these datasets were further divided into subsets according to the song release year: Before the 2000s, from 2000 to 2009, from 2010 topresent.

\section{B. Lyrics Length}

Length of lyrics is a characteristic feature of music and each music genre has its own average music length which could also be used as one of the independent features while predicting a music genre. Music genre's, which divided them further into three time slots, were taken and the total number of words in lyrics were divided with the total number of songs present. For example: Rock music between 2000 to 2009 was taken, total number words in the lyrics was added and divided by the number of songs in this category. This procedure was repeated for all three time slots of each of the five music categories.

\section{Frequently Used Words}

To evaluate the most frequently used words in the fullset of lyrics, we used 'count()' and 'top_n()' function to get the four top words from the dataset which we had cleaned and filtered. Then the words can be sorted in ascending order of their count using 'reorder()' function and we used dplyr library's 'mutate()' to reassign the ordered value to word. Doing this helps ggplot to present the graphs in a clean and effective way.

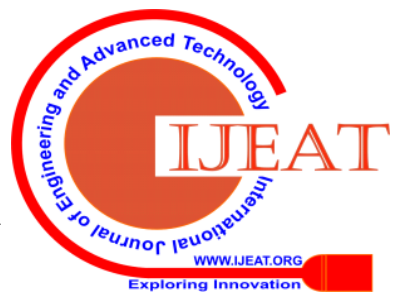




\section{Evolution of Different Music Genres}

Frequently used words of each genre helps to understand the overall emotions which each music carries and how the message and tone have changed over time.

\section{Lexical Diversity}

Lexical Diversity is the representation of a text's vocabulary variation. Higher the diverse vocabulary it contains, greater the lexical diversity of the text. Song vocabulary reflects the number of unique words present in the lyrics of the song. words in the lyrics divided by the total number

of words present in the lyrics. A simple graph with lexical diversity in the $\mathrm{Y}$-axis and year of release in $\mathrm{X}$-axis for a particular music type would help us visualize how the style of song writing has changed over time for that particular song type.

\section{E. Sentimental Analysis}

Sentimental analysis is the process of using Natural Language Processing, computational intelligence and text analysis for text mining and aims to ascertain the opinion and mood of its content. The findings in songs can reflect not only the attitudes of the artist but also the global cultural influences and act as the representation of society and its accepted norms and desires. There are several methods to perform sentimental analysis and we have adopted a predefined lexical dictionary which contains a list of positive and negative words.

The 'tidytext' library in $\mathrm{R}$ includes a dataset called 'sentiments' which has a collection of distinct lexicons and these dictionary words are labelled with a sentiment value of category. While there are three general purpose lexicons widely used: AFINN, BING and NRC [4], we have used BING because it is a simple lexical dictionary with binary labels as positive or negative. Keeping the $\mathrm{X}$-axis as the release year and $\mathrm{Y}$-axis as the sentiment score would show how each genre has evolved over time to listener's preference.

\section{F. Genre Prediction and classification}

In this section, we try to assess how efficient is Natural Language Processing in classifying songs purely based on lyrics. To create our model, we create training and testing tidy data-frames and perform feature engineering to create predictors for our models. This would help us identify the algorithms to use for classification. We then train ourmodels and benchmark the best options. There have been work on music genre classification based on KNN [5], while some few works focus on mood and genre classification together. [6]

To create our predictors based on lyrics, we need to pick features which makes each song unique in its own right. We have already found the most frequently used words and their respective count in each music genre and we select the top ' $n$ ' words of this list where ' $n$ ' is a variable whose optimalvalue depends on the dataset. The choice of $n$ is critical because a low value will not give an optimized result while a high value will lead to overfitting and distort the results. Frequent words common to multiple genres were removed for cleaner and distinct results. This enables us to use genre-specific words in our predictors. We have applied ten times higher weight to explicit words as compared to genre-specific words because explicit words are unique and distinct to each genre. Now these set of genre-specific words were studied for their frequency, average length and repetitions to create our predictors because they are
This can be represented by a graph with average unique

quantitative features. The benchmark used to assess the performance measure of our model is accuracy and time to train. Accuracy is the fraction of correct predictions to total predictions.

\section{III.RESULTS AND DISCUSSION}

In this section we present the results of the methods discussed in the previous section and discuss the interpretation of these results.

\section{A. Lyrics Length}

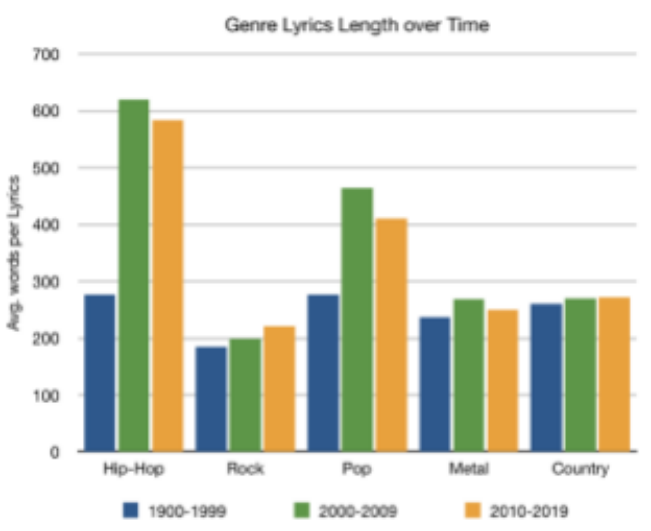

Fig. 1. Lyrics length of different genres over time Fig 1. Shows that Hip-Hop music has the longest songs while rock has the shortest. And while the lyricists of each genre started writing longer songs after the $20^{\text {th }}$ century, there has been trend towards shorter songs in the past decade for each music genre except for country and rock music.

\section{B. Frequenty Used Words}

- $\quad$ Hip-Hop

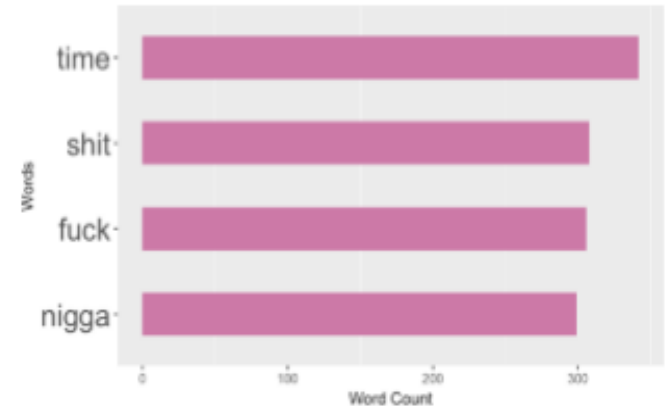

Fig. 2. Most frequently used words in Hip-Hop before the 2000s

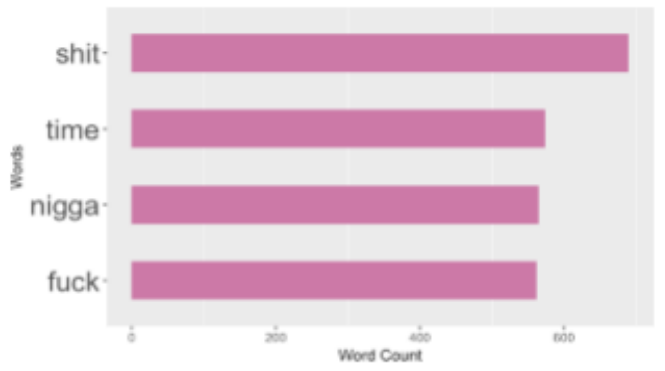

Fig. 3. Most frequently used words in Hip-Hop from 2000 to 2009 


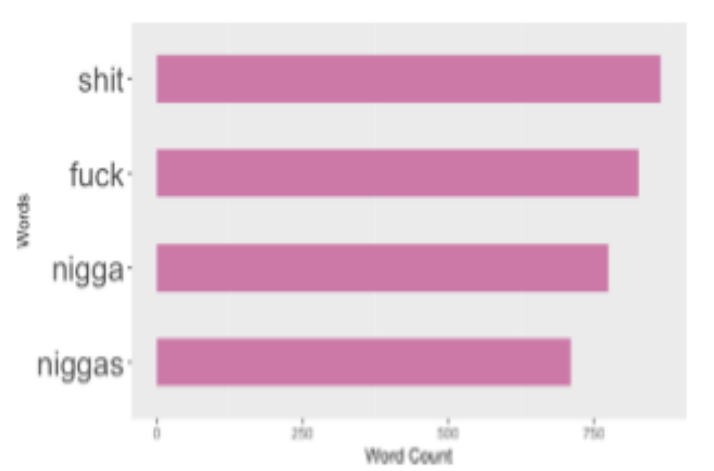

Fig. 4. Most frequently used words in Hip-Hop from 2010 to 2019

Figure 2, 3 and 4 show that Hip-Hop has a heavy use of expletives and its usage has only increased over the years. While Hip-Hop is known to be a channel for its artist to express their frustrations, it is rather disturbing to see the ever- increasing use of profanity and its acceptance by the society.

- Rock

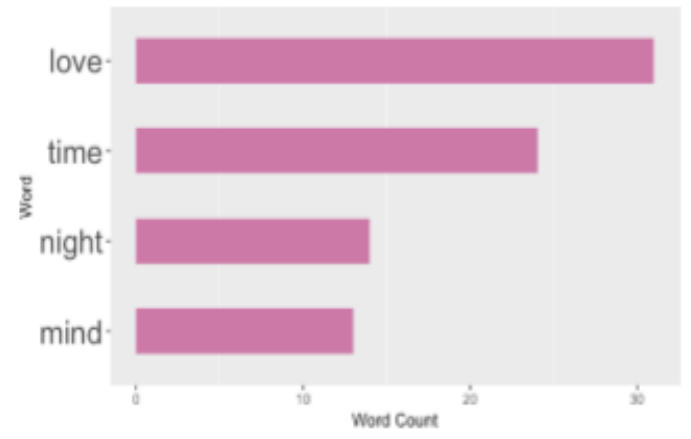

Fig. 5. Most frequently used words in Rock before the 2000s

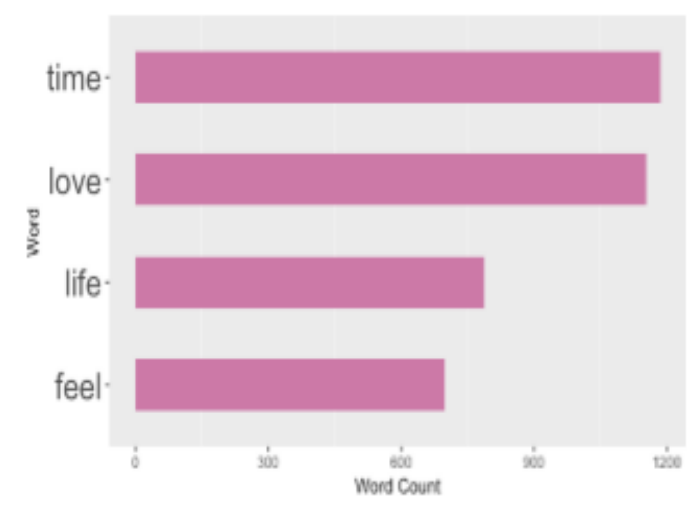

Fig. 6. Most frequently used words in Rock from 2000 to 2009

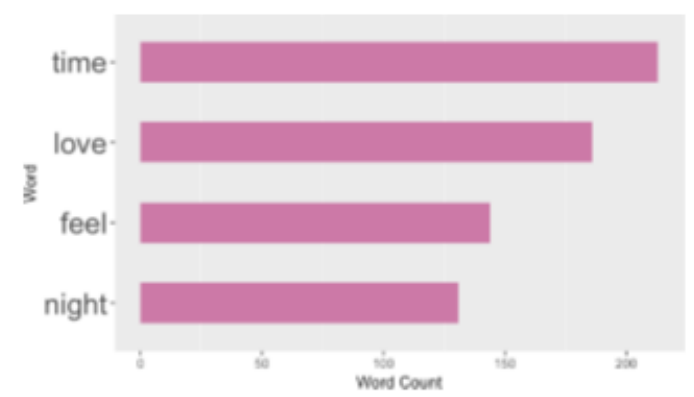

Fig. 7. Pop Most frequently used words in Rock from 2010 to 2019
The lyrics of Rock music is poetic and has a powerful and emotional aura. The lyrics often convey sensations and stories. Although rock has changed extensively since the 1960s, the format and theme have remained the same, as evident from Fig 5, 6 and 7. Lyrics tend to focus on romantic love and other strong emotions.

- Pop

Since it emerged over fifty years ago, pop has been a component of mainstream music and it still plays an important part in people's lives. Fig 8, 9 and 10 show that 'Love' is the predominant topic of pop music and attracts the crowd immediately, especially adolescents and teens. Over the years, Pop has maintained a similar theme of love, girl and feel and has hardly evolved in terms of frequently used words.

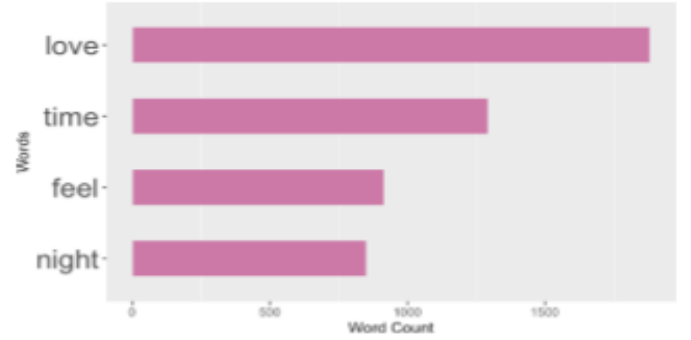

Fig. 8. Most frequently used words in Pop before the 2000s

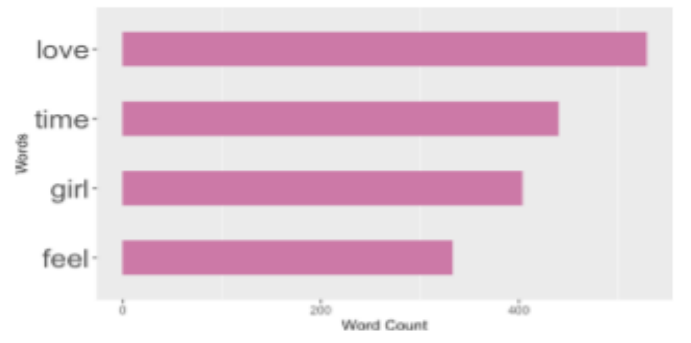

Fig. 9. Most frequently used words in Pop from 2000 to 2009

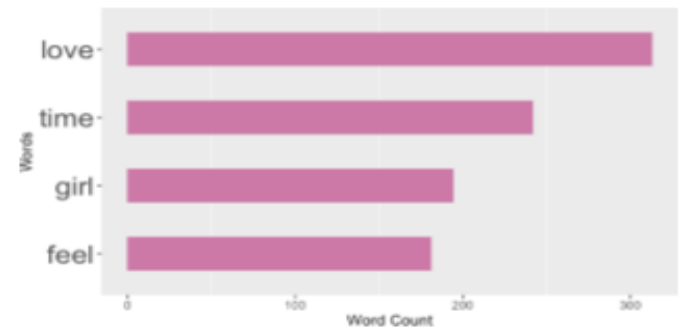

Fig. 10. Most frequenty used words in Pop from 2010 to 2019

- Metal

Fig 11, 12 and 13 show that metal music saw almost no change from 20th century to the first decade of the 21st century with 'time', 'life' and 'world' being the dominant themes. But this genre has now started to evolve and 'life' taking priority over 'time' and new word 'feel' entering the most frequently used words in the lyrics. 


\section{Evolution of Different Music Genres}

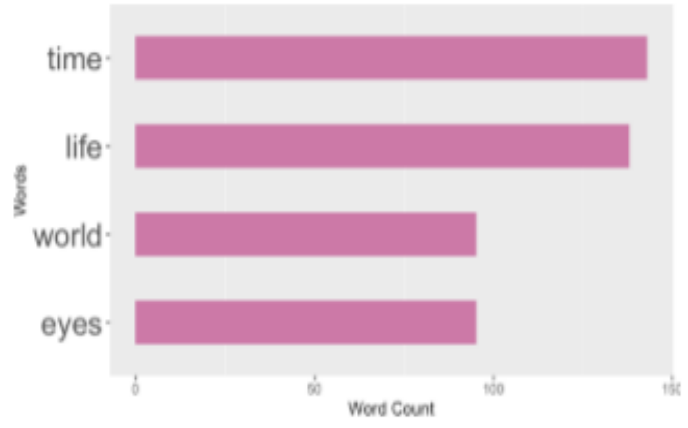

Fig. 11. Most frequenty used words in Metal before the 2000s

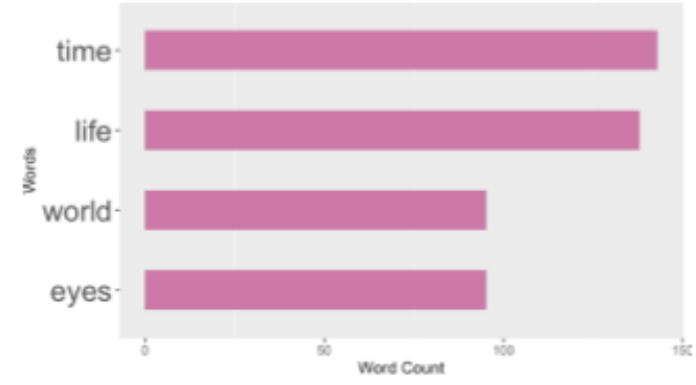

Fig. 12. Most frequently used in Metal from 2000 to 2009

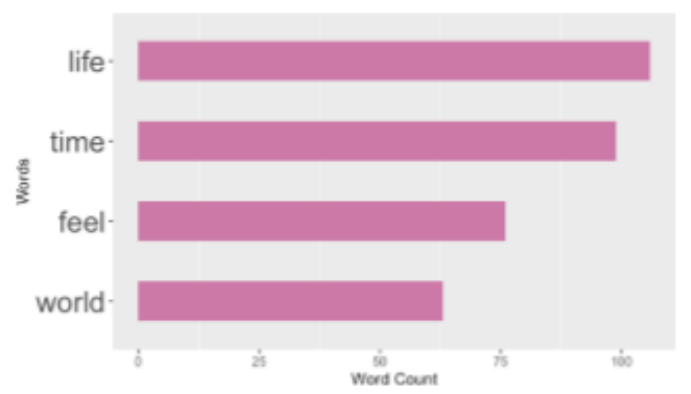

Fig. 13. Most frequently used in Metal from 2010 to 2019

- Country

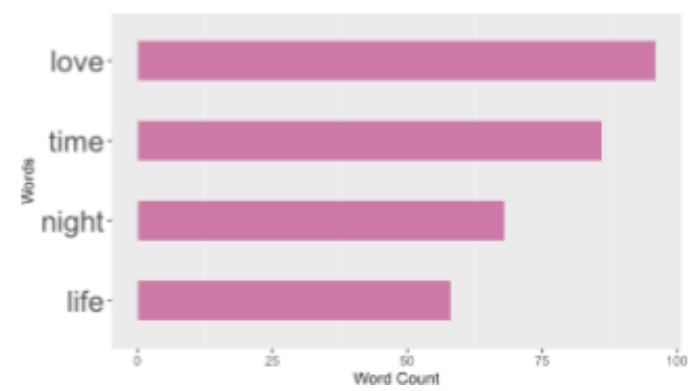

Fig. 14. Most frequently used in Country before the 2000s.

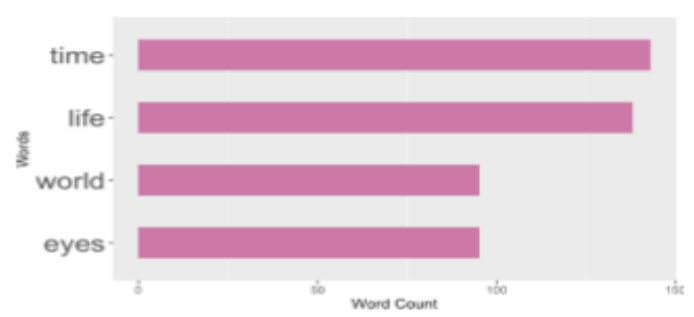

Fig. 15. Most frequently used in Country from 2000 to 2009

Retrieval Number: A1674109119/2019@BEIESP

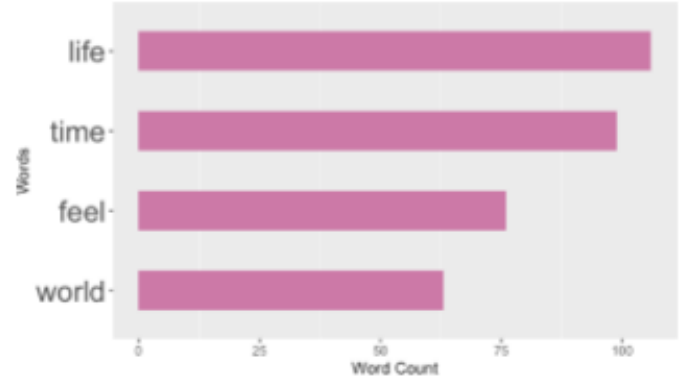

Fig. 16. Most frequently used in Country from 2010 to 2019

From fig 14, 15 and 16, it can be inferred that the most frequently used words in country music before the 2000's were 'love', 'time', 'night' and 'life'. 'Time' replaced 'love' as the most frequently used word between 2000 and 2009. 'Life' was the most frequently occurring word in country songs after 2010. Compared to other genres of music, it is clear that there has been a significant change in the writing style, tone and choice of words in country music as it is the only genre with different highest occurring word in all three time slots. Country song is by far the most dynamic music genre among the rest of the music styles.

\section{Lexical Diversity}

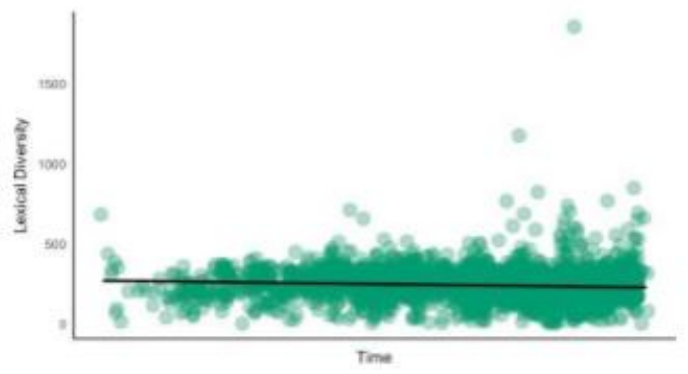

Fig. 17. Lexical diversity of Hip-Hop over time

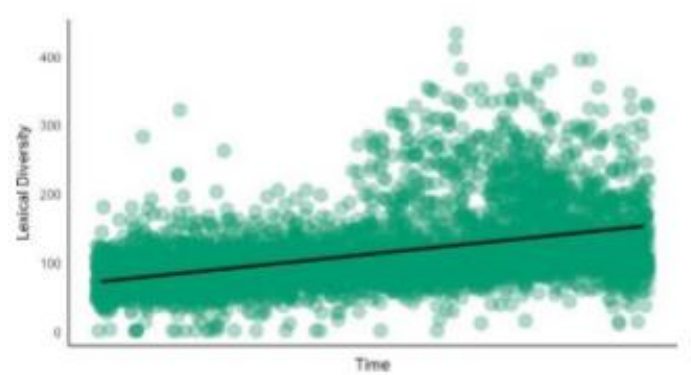

Fig. 18.Lexical diversity of Pop over time

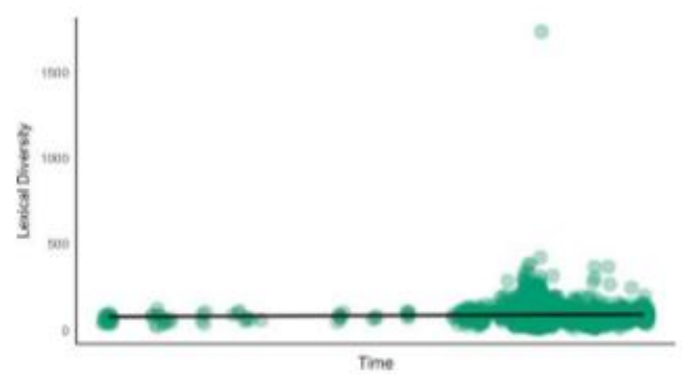

Fig. 19. Lexical diversity of Rock over time

Published By:

Blue Eyes Intelligence Engineering

\& Sciences Publication 


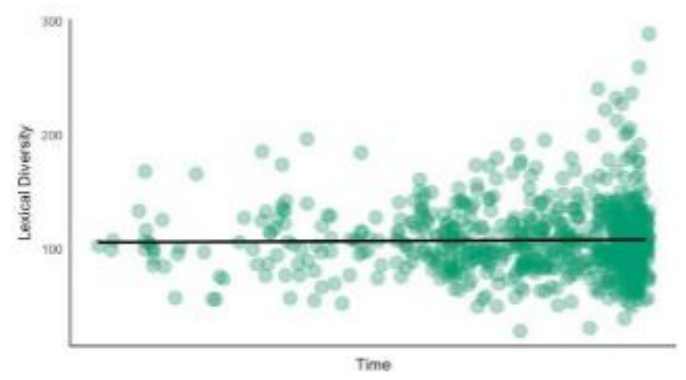

Fig. 20.Lexical diversity of Country over time

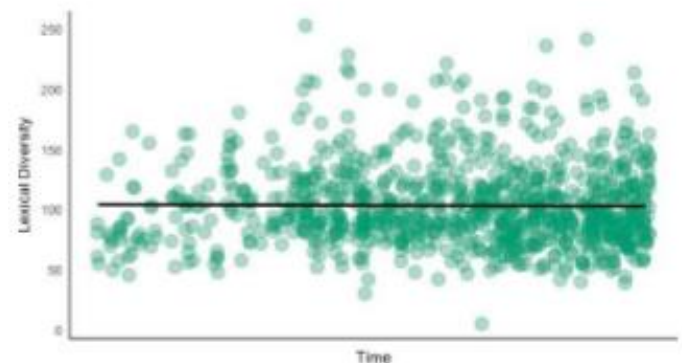

Fig. 21.Lexical diversity of Metal over time

Fig 17 shows that that there has been a slight decrease in the lexical diversity of Hip-Hop music over the years in spite of few songs which released recently having very high lexical diversity. This goes to show that Hip-Hop writers have slowly started using 'repetitions' more in theirsongs.

Fig 18 shows that Pop music has seen an upward trend in terms of lexical diversity and it is an expected result because from Fig 8, 9 and 10, it is seen that Pop music has revolved around the same themes for decades and this must have caused lyricist to use new and varied words to stay relevant and bring a sense of freshness to the audience while also catering to their needs for the same topics.

Fig 19, 20 and 21 show that other music genres: rock, country and metal have remained consistent with their lexical diversity. This shows that lyricists have not felt any need to change repetitions or introduce new words to their writing.

\section{Sentimental Analysis}

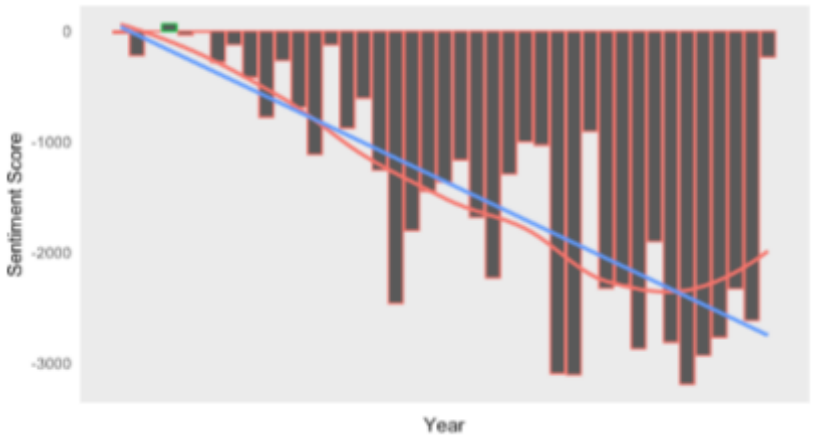

Fig. 22.Sentiment of Hip-Hops songs over time

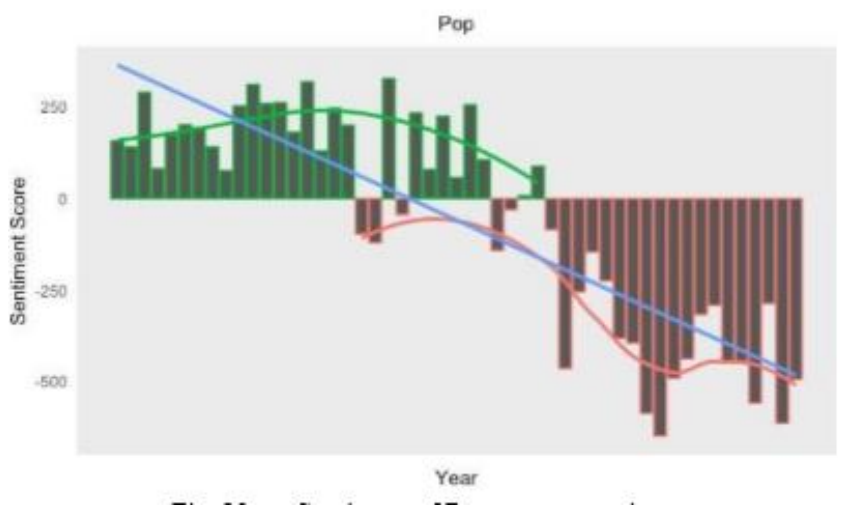

Fig. 23.Sentiment of Pop songs over time

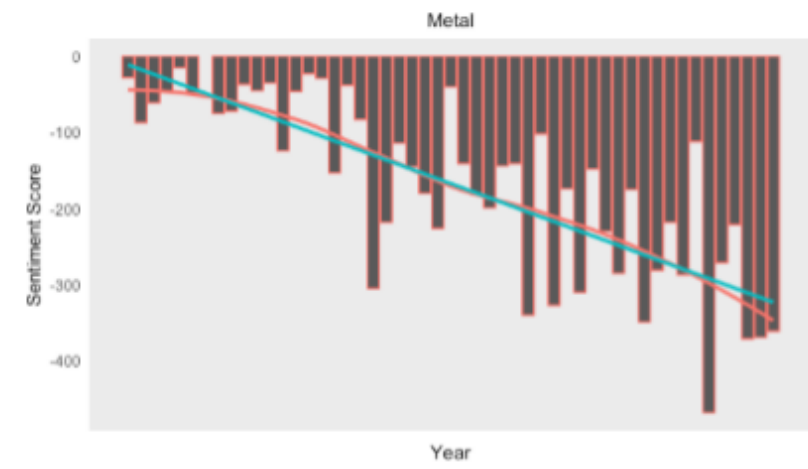

Fig. 24.Sentiment of Metal songs over time

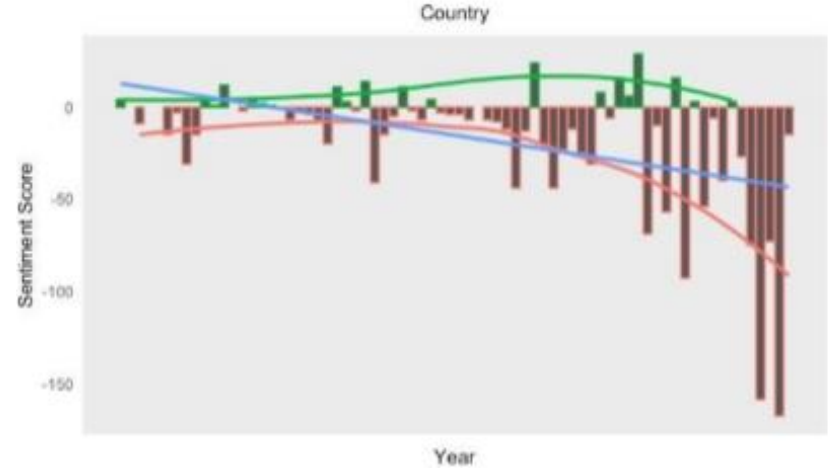

Fig. 25.Sentiment of Country songs over time

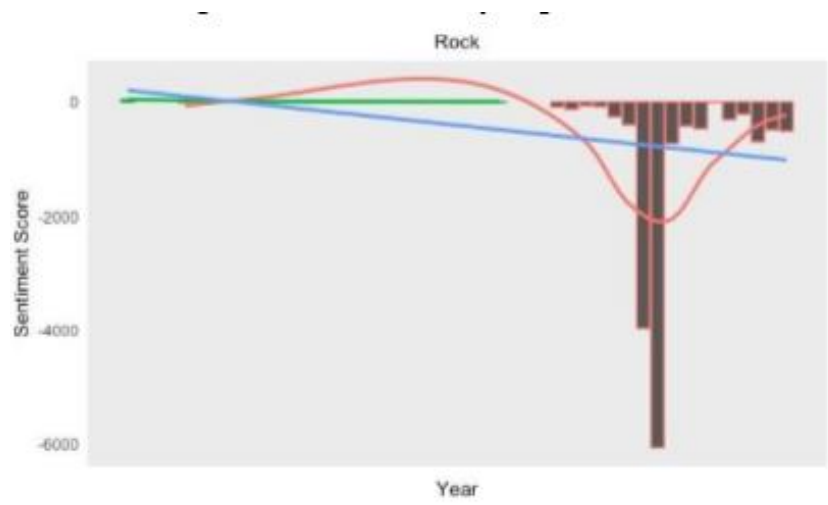

Fig. 26.Sentiment of Rock songs over time

Sentimental Analysis of all music genres shows a negative trend. While genre like Hip-Hop would always have a negative score because of the expletives it uses, other genres like Pop has also slowly evolved into a sentimentally negative genre.

Blue Eyes Intelligence Engineering \& Sciences Publication 


\section{Evolution of Different Music Genres}

The results might indicate societies' attitude towards profanity and expletives, which are thought to be the norm and even appreciated by people. This trend is worrisome considering the omnipresence of music in our lives and the power it has to sub-consciously affect our brain and our actions. This result also acts an alarm for parents who might not be very cautious of their children's choice of music and be unaware of the affects which today's music might have on the impressionable minds of their children.

\section{E. Genre Prediction and Classification}

Fig 27. shows the accuracy of various model with respect to the number of frequently used words or the top_n_words variable discussed in previous section. For our dataset, keeping the variable value at 1500 yielded the highest accuracy for Random Forest with an accuracy of 80.61\%. Random Forest model has consistently shown higher accuracy than other classification models. Keeping a value higher than 1500 leads to overfitting and our accuracy starts decreasing and similarly a value lower than 1500 leads to a result which is not optimized as per our dataset.

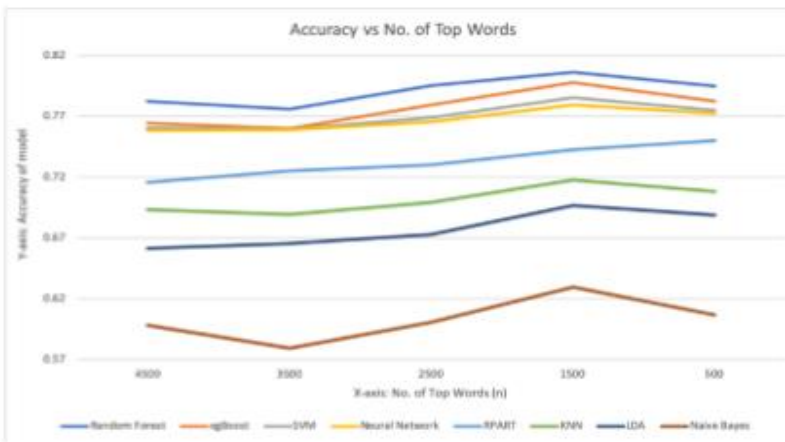

Fig. 27.Accuracy of Various Prediction Model in Genre Classification

TABLE I.

\begin{tabular}{|c|c|c|c|c|c|c|c|c|}
\hline & $\begin{array}{c}\text { Random } \\
\text { Forest }\end{array}$ & $\boldsymbol{x g B o o s t}$ & SVM & $\begin{array}{c}\text { Neural } \\
\text { Network }\end{array}$ & RPART & KNN & LDA & $\begin{array}{c}\text { Nä̈ve } \\
\text { Bayes }\end{array}$ \\
\hline $\mathbf{4 5 0 0}$ & 0.7822 & 0.7645 & 0.7607 & 0.7587 & 0.7159 & 0.6932 & 0.6612 & 0.5983 \\
\hline $\mathbf{3 5 0 0}$ & 0.7758 & 0.7602 & 0.7594 & 0.7589 & 0.7249 & 0.6895 & 0.6655 & 0.5793 \\
\hline $\mathbf{2 5 0 0}$ & 0.7952 & 0.7794 & 0.7687 & 0.7655 & 0.7299 & 0.6994 & 0.6731 & 0.6005 \\
\hline $\mathbf{1 5 0 0}$ & 0.8061 & 0.7976 & 0.7855 & 0.7792 & 0.7428 & 0.7177 & 0.6969 & 0.6294 \\
\hline $\mathbf{5 0 0}$ & 0.7948 & 0.7826 & 0.7751 & 0.7723 & 0.7498 & 0.7081 & 0.6889 & 0.6067 \\
\hline
\end{tabular}

Fig. 28. Classification Model Accuracy vs Top N words

\section{CONCLUSION AND FUTURE WORK}

In this paper, we have discussed about the various parameters like lyrics length, frequently used words, lexical diversity and sentimental analysis of five music genres: Pop, Hip-Hop, Rock, Country and Metal over several decades. Our study has shown how these music styles have evolved and what can we expect from them in the future. While most lyricist started writing longer songs in the first decade of $21^{\text {st }}$ century, they are now writing songs which are ever so slightly shorter. Hip-Hop is filled with expletives and Pop music has been consistent with its theme of love since the beginning and has not evolved much with respect to frequently occurring words. Pop music is the only genre which has seen an increase in lexical diversity and this might be attributed to the constant theme which needs to be conveyed differently to maintain people's interest. The results of the sentimental analysis done on each genre is worrisome as it shows music of all genres becoming more and more negative. Eight classification models were used to classify music genres based on lyrical data and Random Forest fared better than the rest. Keeping the top $\mathrm{N}$ words value at 1500 gave us the best results.

In our future work, we plan to improve upon the classifier models and include more predictor variables to help the models predict better. Though genre classification solely based on lyrics gave an impressive accuracy of $80.61 \%$ for Random Forest, inclusion of more predictors can surely increase this accuracy. A comparative study can also be done on the prediction accuracy of lyrics based and music-based classification [7] of genres.

\section{REFERENCES}

1. Sam Isenberg, 'Lyrics Classifier', (2018), GitHub Repository.

2. Available: https://github.com/saisenberg/lyrics-classifier

3. Charts, Trending on Genius. Available: https://genius.com/

4. Gyanendra Mishra, '380,000+ lyrics from MetroLyrics', 2016 Available:https://www.kaggle.com/gyani95/380000-lyrics-frommetrolyrics

5. Julia Silge and David Robinson, "Text Mining with R, A tidy Approach", Chapter 2: 'Sentimental Analysis with tidy data'. 201908-05. Available:https://www.tidytextmining.com/sentiment.html

6. Tamatjita, E. N. (2016). Comparison of Music Genre Classification Using Nearest Centroid Classifier and k-Nearest Neighbours, (November), 118-123.

7. Shakya, A., Gurung, B., Thapa, M. S., Rai, M., \& Joshi, B. (2017). Music classification based on genre and mood. In Communications in Computer and Information Science (Vol. 776, pp. 168-183). https://doi.org/10.1007/978-981-10-6430-2_14

8. Baniya, B. K., \& Hong, C. S. (2015). Music Mood Classification using Reduced Audio Features. 한국정보과학회 학술발표논문집, 915-917.

\section{AUTHORS PROFILE}

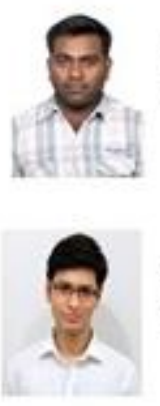

Dr. Selvakumar Kamalnathan is currently working as an Associate Professor in the School of Computer Science and Engineering(SCOPE) in VIT, Vellore.

Yash Mishra is currently pursuing B-Tech in Computer Science Engineering( $4^{\mathrm{b}}$ Year) in VIT University, Vellore.

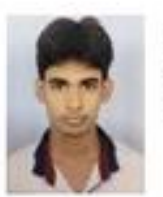

Vijay Kumawat is eurrently pursuing B-Tech in Computer Science Engineering( $4^{\text {th }}$ Year) in VIT University, Vellore.

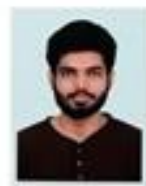

Vaibhav Bangwal is currently pursuing B-Tech in Computer Science Engineering ( $4^{\mathrm{t}}$ Year) in VIT University, Vellore. 